\title{
LDPC Codes for Interference Channels in the Primary Decodes Cognitive Regime
}

\author{
Mahdi Shakiba-Herfeh, Student Member, IEEE, A. Korhan Tanc ${ }^{\circledR}$, Member, IEEE, \\ and Tolga M. Duman ${ }^{\circledR}$, Fellow, IEEE
}

\begin{abstract}
We investigate cognitive interference channels (CICs), a variant of two-user ICs in which one of the transmitters (called the cognitive transmitter) has non-causal knowledge of the other's (private user's) message. Prompted by the information theoretical results, we design an explicit coding scheme for CIC in the primary decodes cognitive regime. We present a novel joint decoder and design low-density parity-check codes for this set-up. Simulation results demonstrate that the proposed joint decoder and the designed codes outperform the conventional maximum ratio combining type decoder and the point-to-point optimal codes, respectively.
\end{abstract}

Index Terms-Low-density parity-check codes, cognitive interference channel, code design, iterative joint decoding.

\section{INTRODUCTION}

C OGNITIVE interference channel (CIC) (motivated by cognitive radio systems [1]) is a type of interference channel with primary and cognitive transmitter-receiver pairs, where the cognitive transmitter has non-causal knowledge of the primary message. The capacity region of CIC is not known in general, however, the "primary decodes cognitive" regime is one of the regimes with a known capacity region [2]. Information theoretical results show that, in this regime, the optimal strategy is dirty paper coding (DPC) against the interference caused by the primary message at the cognitive receiver. At the receivers' side, the cognitive receiver decodes only its own message while the primary receiver decodes both messages.

As a complementary study to the existing information theoretical works [2]-[4], in this letter, we develop channel coding solutions for cognitive interference channels in the primary decodes cognitive regime. To the best of our knowledge, [5] is the only work in the literature on explicit channel coding solutions for this challenging problem. In [5], the cognitive transmitter utilizes DPC against the interference, and the primary receiver combines three different transformations of the channel observation using a maximum ratio combiner (MRC). The output of MRC is fed to a Bahl-Cocke-Jelinek-Raviv (BCJR) decoder, which works in parallel with two single-user decoders. We make the observation that the method of combining the transformations of the channel observation is not

Manuscript received February 24, 2019; accepted April 5, 2019. Date of publication April 16, 2019; date of current version August 21, 2019. This work was supported by the Scientific and Technological Research Council of Turkey (TUBITAK) under Grant 114E601. The associate editor coordinating the review of this paper and approving it for publication was W. Zhang. (Corresponding author: A. Korhan Tanc.)

M. Shakiba-Herfeh and T. M. Duman are with the Department of Electrical and Electronics Engineering, Bilkent University, 06800 Ankara, Turkey (e-mail: herfeh@ee.bilkent.edu.tr; duman@ee.bilkent.edu.tr).

A. K. Tanc is with the Department of Biomedical Engineering, Biruni University, 34020 Istanbul, Turkey (e-mail: atanc@biruni.edu.tr).

Digital Object Identifier 10.1109/LWC.2019.2911499 optimal and may degrade the performance of the receiver, and propose a new structure for the primary receiver, in which the primary and cognitive messages are decoded in parallel with the aid of an advanced state node decoder. Due to their promising performance in multiuser communications [6]-[8], we consider low-density parity-check (LDPC) codes as the channel coding solution and develop a code design procedure as well. Basically, we extend the earlier fading IC code design approach of [6] to the new set-up of Gaussian CIC incorporating a cognitive transmitter with an additional DPC encoder against the known interference, and a primary receiver with additional modulo blocks and BCJR decoder. We also optimize scaling factors for the primary receiver and we obtain a stability condition for the iterative decoder. Simulation results demonstrate that the performance of the proposed receiver surpasses that of the approach developed in [5]. Moreover, our designed codes outperform the point-to-point (P2P) optimal ones and the previously reported irregular repeat accumulate (IRA) coding results.

The letter is organized as follows. In Section II, we describe the system model. In Section III, we present the encoding and decoding structures for a CIC in the primary decodes cognitive regime. Assuming LDPC code ensembles, we derive a stability condition and introduce a code optimization procedure in Section IV. In Section V, we provide the newly achieved rate pairs and simulation based error rate results. Finally, we conclude the letter in Section VI.

\section{SYSTEM MODEL}

A two user Gaussian CIC is described by the following input-output relationship [2] $Y_{1}=X_{1}+a X_{2}+z_{1}$ and $Y_{2}=b X_{1}+X_{2}+z_{2}$ where $X_{i}$ is the transmitted signal from the cognitive $(i=1)$ and primary $(i=2)$ transmitters. $X_{i}$ satisfies the power constraint $E\left[X_{i}^{2}\right] \leq P_{i}$, and $z_{i}$ represents the Gaussian noise component at the receiver $i$ with zero mean and variance $N_{i}$, for $i=1,2$. The channel gains are real and assumed to be known at all the terminals. In addition $b>0$.

Armed with a non-causal knowledge of the primary message, the cognitive transmitter can boost the primary user's transmission via superposition coding, i.e., the transmitted signals are $X_{1}=\sqrt{\alpha P_{1}} X_{c}+\sqrt{(1-\alpha) P_{1}} X_{p}$ and $X_{2}=\sqrt{P_{2}} X_{p}$ where $X_{c}$ and $X_{p}$ denote the modulated cognitive and primary codewords, respectively. Notice that $\alpha \in(0,1]$ is the power sharing parameter. Hence the received signals at the cognitive and primary receivers can be represented as follows

$$
\begin{aligned}
& Y_{1}=\sqrt{\alpha P_{1}} X_{c}+\left(a+\sqrt{\bar{\alpha} P_{1} / P_{2}}\right) \sqrt{P_{2}} X_{p}+z_{1}, \\
& Y_{2}=b \sqrt{\alpha P_{1}} X_{c}+\left(1+b \sqrt{\bar{\alpha} P_{1} / P_{2}}\right) \sqrt{P_{2}} X_{p}+z_{2},
\end{aligned}
$$

where $\bar{\alpha}=1-\alpha$. We utilize irregular LDPC codes for transmission and express the variable node (check node) degree 


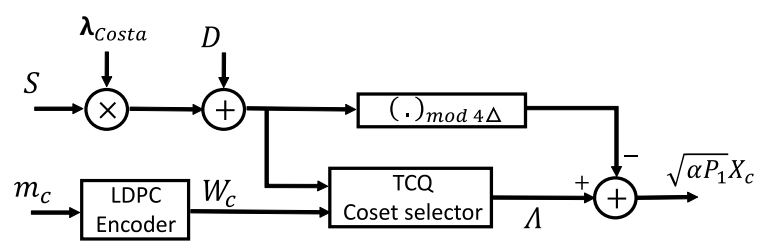

(a)

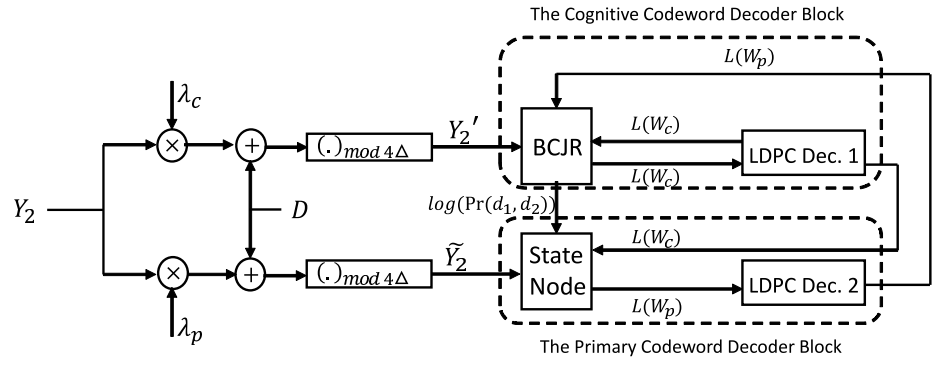

(b)

Fig. 1. (a) The block diagram of the DPC encoder, (b) The block diagram of the proposed receiver.

distribution of the code ensemble as $\lambda(x)=\sum_{i=2}^{d_{v}} \lambda_{i} x^{i-1}$ $\left(\rho(x)=\sum_{i=2}^{d_{c}} \rho_{i} x^{i-1}\right)$, where $d_{v}\left(d_{c}\right)$ is the maximum degree of variable nodes (check nodes).

\section{ENCODER AND DECODER SCHEMES}

The block diagram of the DPC encoder is illustrated in Fig. 1(a). Here $S$ is the interference signal observed at the cognitive receiver given by $S=\left(a+\sqrt{\bar{\alpha} P_{1} / P_{2}}\right) \sqrt{P_{2}} X_{p}$, where the primary message $m_{p}$ is LDPC encoded and binary phase shift keying (BPSK) modulated ( 0 and 1 are mapped to +1 and -1 , respectively) to form $X_{p}$. This signal is scaled by the Costa scaling factor $\lambda_{\text {Costa }}=\alpha P_{1} /\left(\alpha P_{1}+N_{1}\right)$ and added to a pseudo-random dither sequence $D$. The resulting signal is forwarded to a trellis coded quantization (TCQ) unit. The cognitive message $m_{c}$ is encoded by the binary LDPC encoder, and the encoded message $W_{c}$ is fed to the TCQ unit. This unit first quantizes $\lambda S+D$ using Viterbi algorithm, where the trellis is specified by a rate $\frac{1}{2}$ convolutional code, using a modulo $4 \Delta$ distance metric. Here $\Delta$ is the parameter used to adjust the transmit power. Then the resulting sequence is shifted by $\pm \delta / 2$ depending on the value of $W_{c}$. Here we note that instead of the conventional modulo operation, we consider a modification for which the output is in the range of $\left[-\frac{K}{2}, \frac{K}{2}\right)$, where $K$ is the divisor. This modification does not cause any change on the system performance. However, with the new form, the modulo P2P channel satisfies outputsymmetry, which simplifies the derivation of the stability condition. It follows that the elements of TCQ codeword are in the set $A=\{-2 \Delta-\delta / 2,-2 \Delta+\delta / 2,-\Delta-\delta / 2,-\Delta+\delta / 2$, $-\delta / 2, \delta / 2, \Delta-\delta / 2, \Delta+\delta / 2\}$, and the modulated cognitive codeword is obtained as

$$
\begin{aligned}
\sqrt{\alpha P_{1}} X_{c} & =\left[\lambda S+D, W_{c}\right]_{T C Q}-(\lambda S+D)_{\bmod 4 \Delta}, \\
& =\Lambda-(\lambda S+D)_{\bmod 4 \Delta},
\end{aligned}
$$

where $T C Q$ subscript denotes the encoding by TCQ.

At the cognitive receiver, the following transformation is applied:

$$
\begin{aligned}
Y_{1}^{\prime} & =\left(\lambda_{\text {Costa }} Y_{1}+D\right)_{\text {mod4 } 4} \\
& =(\Lambda+\underbrace{\left(\lambda_{\text {Costa }}-1\right) \sqrt{\alpha P_{1}} X_{c}+\lambda_{\text {Costa }} z_{1}}_{\text {noise } z_{1}^{\prime}})_{\text {mod } 4 \Delta},
\end{aligned}
$$

which is equivalent to the P2P transmission of codeword $\Lambda$ over a modulo additive white Gaussian noise (AWGN) channel. Then the modulated cognitive message $X_{c}$ is decoded using an iterative decoding procedure where soft-information exchange takes place between the two decoders. The first decoder is the BCJR decoder for the TCQ, and the second one is the component LDPC decoder employing belief-propagation (BP). Extensive numerical experiments show that the distribution of $X_{c}$ is very close to Gaussian. Hence, as also indicated in [5], the noise $z_{1}^{\prime}$ is approximately Gaussian. For the regime under consideration, the primary receiver decodes both primary and cognitive messages. As an alternative to the joint decoder utilizing MRC in [5], we propose a novel joint decoder with a state node as depicted by Fig. 1(b). In what follows each decoding block is explained in detail.

1) The Cognitive Codeword Decoder: As depicted in Fig. 1(b), this decoder block consists of a BCJR decoder connected to a component LDPC decoder where $\lambda_{c}$ is the scaling factor of the block. We consider a transformation of $Y_{2}$ such that

$$
\begin{aligned}
Y_{2}^{\prime}= & \left(\lambda_{c} Y_{2}+D\right)_{\bmod 4 \Delta}=(\Lambda+\underbrace{\left(\lambda_{c} h_{p}-\lambda_{\text {Costa }} h_{s}\right) X_{p}}_{\text {interference }} \\
& +\underbrace{\left(\lambda_{c} b-1\right) \sqrt{\alpha P_{1}} X_{c}+\lambda_{c} z_{2}}_{\text {noise } z_{2}^{\prime}})_{\bmod 4 \Delta},
\end{aligned}
$$

where $h_{s}$ and $h_{p}$ are the coefficients determining the contributions of $X_{p}$ to the signals $Y_{1}$ and $Y_{2}$, respectively. $W_{p}$ is the primary codeword, $d_{1}$ and $d_{2}$ are the quantized bits corresponding to $\lambda S+D$. The noise signal $z_{2}^{\prime}$ is approximately Gaussian with zero mean and variance $P_{z_{2}^{\prime}}=\left(\lambda_{c} b-1\right)^{2} \alpha P_{1}$ $+\lambda_{c}^{2} N_{2}$. In order to minimize the sum of interference and noise power, $\lambda_{c}$ is optimized as

$$
\hat{\lambda}_{c}=\underset{\lambda_{c} \in \mathbb{R}}{\arg \min }\left(\lambda_{c} h_{p}-\lambda_{\text {Costa }} h_{s}\right)^{2}+P_{z_{2}^{\prime}} .
$$

The BCJR algorithm is used to calculate the extrinsic loglikelihood ratios (LLRs) of the cognitive codewords represented by $L\left(W_{c}\right)$. In addition, it calculates the logarithm of the probabilities for the bits $\left(d_{1}, d_{2}\right)$ which are sent to the $W_{p}$ decoder. On the other hand, the component LDPC decoder improves the LLRs of the cognitive codewords and supplies them to the $W_{p}$ decoder.

2) The Primary Codeword Decoder: As depicted in Fig. 1(b), this decoder block consists of a state node connected to a component LDPC decoder, where $\lambda_{p}$ is the scaling factor of the block. The state node updates $L\left(W_{p}\right)$ using the transformed received signal $\tilde{Y}_{2}$ and $L\left(W_{c}\right)$ coming from the DPC decoder, whereas the component LDPC decoder decodes the 
primary message. $\tilde{Y}_{2}$ is simply defined as

$$
\begin{aligned}
\tilde{Y}_{2}= & \left(\lambda_{p} Y_{2}+D\right)_{\bmod 4 \Delta}=(\Lambda+\underbrace{\left(\lambda_{p} h_{p}-\lambda_{\text {Costa }} h_{s}\right) X_{p}}_{\text {interested message. }} \\
& +\underbrace{\left(\lambda_{p} b-1\right) \sqrt{\alpha P_{1}} X_{c}+\lambda_{p} z_{2}}_{\text {noise } \tilde{z}_{2}})_{\bmod 4 \Delta} .
\end{aligned}
$$

At the state node, $L\left(W_{p}\right)$ is calculated as

$$
L\left(W_{p}[i]\right)=\log \left(\frac{f_{\tilde{Y}_{2}}\left(\tilde{Y}_{2}[i] \mid W_{p}[i]=0\right)}{f_{\tilde{Y}_{2}}\left(\tilde{Y}_{2}[i] \mid W_{p}[i]=1\right)}\right),
$$

where the conditional probability density functions (PDFs) are calculated by marginalizing over $\Lambda$ similar to the procedure described in [8]. In order to maximize the signal-to-noise ratio in (6), we optimize $\lambda_{p}$ as

$$
\hat{\lambda}_{p}=\underset{\lambda_{p} \in \mathbb{R}}{\arg \max } \frac{\left(\lambda_{p} h_{p}-\lambda_{\text {Costa }} h_{s}\right)^{2}}{\left(\lambda_{p} b-1\right)^{2} \alpha P_{1}+\lambda_{p}^{2} N_{2}} .
$$

We note that, while $\lambda_{c}$ is optimized by minimizing interference and noise powers, $\lambda_{p}$ is optimized under the assumption that the interference signal $\Lambda$ is known and can be cancelled out perfectly. While this is a suboptimal approach, it simplifies the parameter optimization considerably.

\section{Performance Analysis}

\section{A. Stability Condition}

Stability analysis examines the convergence of the iterative decoder under the assumption that the error probability is very small. In the following, we derive a stability condition for the cognitive message decoded at both receivers, and for the primary message decoded at the primary receiver. In the derivations, we assume asymptotic analysis of LDPC codes and error-free BCJR decoding on the TCQ trellis.

1) The Cognitive Message: We assume that the interference signal and the convolutional code (coset selector) have been completely decoded and cancelled out from the received signals. Under this assumption, (3) and (4) turn into $Y_{1}^{\prime}=$ $\left(\delta / 2-\delta W_{c}+z_{1}^{\prime}\right)_{\bmod 4 \Delta}$ and $Y_{2}^{\prime}=\left(\delta / 2-\delta W_{c}+z_{2}^{\prime}\right)_{\bmod 4 \Delta}$, respectively. These modified channels are actually modulo $\mathrm{P} 2 \mathrm{P}$ channels. In the Appendix, the Bhattacharyya constant of a modulo P2P channel with BPSK modulation is derived. The overall stability condition for the cognitive LDPC code ensemble is that the product of derivatives $\lambda^{\prime}(0)$ and $\rho^{\prime}(1)$ should be smaller than the minimum of the two Bhattacharyya constants derived from the analysis for the cognitive and primary receivers, respectively.

2) The Primary Message: We derive the stability condition for the primary code ensemble under the assumption that the convolutional code and cognitive message are completely decoded and cancelled out. Hence, the channel in (6) turns into $\tilde{Y}_{2}=\left(\lambda_{p} h_{p} X_{p}-\lambda_{\text {Costa }} h_{s} X_{p}+\tilde{z}_{2}\right)_{\bmod 4 \Delta}$. The stability condition of the primary code ensemble is obtained similarly by utilizing the Bhattacharyya constant of this modulo P2P channel.

\section{B. LDPC Code Design}

In the code design, we aim to find the best rate pairs for which reliable communication can take place for the given set of channel parameters. We employ the recursive systematic

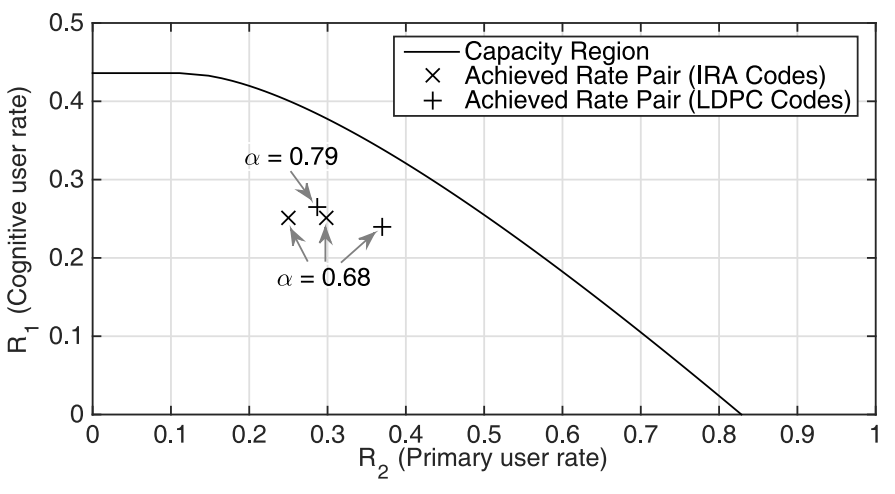

Fig. 2. The channel capacity and the achieved points.

convolutional (RSC) code $[625,242]^{1}$ in octal form for the TCQ [10]. To optimize the LDPC code ensembles, we utilize small perturbations to find codes with higher rates gradually. The decoding thresholds of the candidate ensembles are examined by an extrinsic information transfer (EXIT) chart analysis powered by the stability condition, where the latter is checked for eliminating error floors. While utilizing the EXIT chart analysis, we make a Gaussianity assumption for the LLRs exchanged among the blocks, except for the LLRs incoming to the state node. Due to the non-linearity of the state node function, we model the links of the incoming LLRs to the state node as binary erasure channels [7]. We calculate the mutual information of the LLRs outgoing from the BCJR decoder by utilizing a look-up table which is constructed using Monte-Carlo simulations.

If the ensembles satisfy the stability condition, and if their decoding tunnels are open, which is examined using the EXIT chart analysis, we consider them as admissible. The process of searching for new ensembles continues until the admissible code ensembles cannot be further improved in terms of rate.

\section{Numerical Results}

We first consider the same example in [5] with parameters $P_{1}=1.1, P_{2}=1, N_{1}=1.325, N_{2}=3.15, a=-1.2$, $b=1.53$ and assume an MRC type decoding at the primary receiver. Fig. 2 shows the capacity region and the achieved rate pair reported in [5] with off-the-shelf IRA codes with 40k block length at a bit error rate of $10^{-5}$. Assuming the same block length and bit error rate, our code design results in rate improvements, e.g., $(0.239,0.37)$ and $(0.265,0.288)$ rate pairs can be achieved with the proposed joint decoder structure. Table I shows the designed LDPC degree distributions. We also improve the performance obtained with IRA codes by utilizing the proposed decoder and achieve $(0.25,0.298)$ rate pair assuming off-the-shelf degree distributions. Note that the newly designed LDPC codes offer a better performance.

Next we consider an instance of CIC with parameters $P_{1}=$ 1.1, $P_{2}=1, N_{1}=1.325, N_{2}=3.15, a=-0.38, b=2$ and $\alpha=0.68$. For this example, we perform LDPC code design for both the proposed joint decoder and the MRC-type decoder. We provide the resulting degree distributions in Table I. In Fig. 3, we illustrate the error-rate performance of the designed LDPC codes for the primary message with block length 40k. In the same figure, we also show the performance of $\mathrm{P} 2 \mathrm{P}$ optimal codes with the same rate and block length. We observe that, at a bit error rate of $10^{-4}$, the advantage of joint decoding is

\footnotetext{
${ }^{1}$ We choose this generator polynomial owing to its good performance in DPC [9].
} 
TABLE I

OPTIMIZED LDPC DEGREE DISTRIBUTIONS

\begin{tabular}{|c|c|c|c|c|c|c|c|c|c|c|c|c|c|c|}
\hline Mes & $\mathrm{R}$ & $\lambda_{2}$ & $\lambda_{3}$ & $\lambda_{4}$ & $\lambda_{6}$ & $\lambda_{7}$ & $\lambda_{12}$ & $\lambda_{13}$ & $\lambda_{25}$ & $\lambda_{100}$ & $\lambda_{200}$ & $\rho_{2}$ & $\rho_{6}$ & $\rho_{7}$ \\
\hline$W_{c}$ (Fig. 2) & 239 & 2376 & 1258 & 1279 & 0260 & 0748 & .0141 & .0533 & .0288 & 0.0954 & 0.2163 & 0.0126 & 0.7228 & 0.2646 \\
\hline$W_{c}(1$ & 265 & 2443 & 7 & 3 & 0 & 0 & 0072 & 96 & .0301 & 87 & 0.2 & & & 0.2494 \\
\hline$W_{p}$ (Fig. 2) & 288 & 3209 & 6 & 2 & 7 & & & & 51 & & 4 & & 0 & 0.2669 \\
\hline$W_{c}$ (Fig. & 239 & 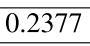 & 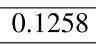 & 1279 & 260 & .0748 & 41 & 0.0533 & 28 & & 63 & & & 46 \\
\hline Message & $\mathrm{R}$ & $\overline{\bar{\lambda}}$ & $\overline{\bar{\lambda}}$ & $\lambda_{4}$ & $\lambda_{6}$ & $x_{1}$ & & 年 & 200 & & & & & \\
\hline (g. 2) & 370 & 2407 & 0715 & 100 & 375 & 0749 & $011^{2}+2$ & .0112 & .2367 & .0947 & 0.0330 & .0035 & 0.7395 & 0.2570 \\
\hline$W_{p}$ (Fig. 3) & 394 & 3162 & 1448 & 0868 & 01 & 14 & 0.0241 & 0.0004 & 0.1426 & 0.0944 & 0.1692 & 0.0420 & 0.6893 & 0.2688 \\
\hline$W_{p}$ (Fig. 3) & 0.394 & 0.2299 & 0.1238 & 0.1868 & 0.0227 & 0.0222 & 0.0521 & 0.0018 & 0.3235 & 0.0215 & 0.0159 & 0.0016 & 0.7062 & 0.2922 \\
\hline
\end{tabular}

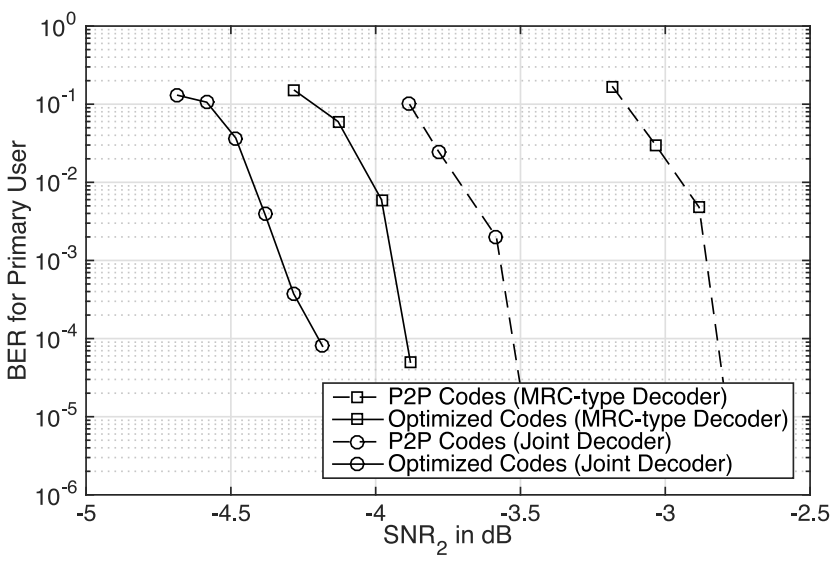

Fig. 3. Finite block length decoding results. $S N R_{i}$ is defined as $P_{i} / N_{i}$ and $S N R_{2} / S N R_{1}$ is kept fixed.

at least $0.3 \mathrm{~dB}$. Furthermore, the specific code design provides an improvement of at least $0.7 \mathrm{~dB}$. However, we note that, the joint decoding advantage depends on the channel parameters, i.e., there exist channel parameters for which the MRC-type decoder surpasses the proposed joint decoder.

\section{CONCLUSION}

We introduce an explicit coding scheme for Gaussian CICs in the primary decodes cognitive regime. We propose a novel joint decoding scheme and develop an LDPC code design procedure. Simulation results show that the newly designed LDPC codes provide rate improvements over the previously reported IRA codes. Furthermore, the proposed decoding scheme provides improvements over the conventional MRC type decoding, and further improvements can be attained with the new LDPC codes compared to the P2P optimal ones.

\section{APPENDIX}

\section{DERIVATION OF THE BHATTACHARYYA CONSTANT FOR THE MODULO CHANNEL}

In this Appendix, we consider the modulo channel $f_{Y \mid x}(y) \sim(h x+n)_{\bmod K}$, where $x \in\{-1,1\}$ and $n \sim$ $\mathcal{N}\left(0, \sigma^{2}\right)$. The LLR of the observed signal which is the logarithm of $P(y \mid x=1) / P(y \mid x=-1)$ can be obtained as

$$
L L R(y)=\frac{2 y h}{\sigma^{2}}+\log \left(\frac{\sum_{l \in \mathbb{Z}} \exp \left(-\frac{l^{2} K^{2}-2 l(y-h) K}{2 \sigma^{2}}\right)}{\sum_{l \in \mathbb{Z}} \exp \left(-\frac{l^{2} K^{2}-2 l(y+h) K}{2 \sigma^{2}}\right)}\right) .
$$

In order to find the Bhattacharyya constant of the channel $\mathcal{B}\left(P_{L}\right)$, we calculate

$\mathcal{B}\left(P_{L}\right)=\int_{\mathbb{R}} P_{L}(l) \exp \left(-\frac{l}{2}\right) d l=\int_{\mathbb{R}} P_{Y}(y) \exp \left(-\frac{L(y)}{2}\right) d y$,

where $P_{L}(l)$ is the $L L R$-density of the channel output and $P_{Y}(y)$ is the $Y$-density of the channel. By using the symmetry of the channel, we utilize

$$
P_{Y}(y \mid x=1)=\frac{1}{\sqrt{2 \pi \sigma^{2}}} \sum_{l \in \mathbb{Z}} \exp \left(-\frac{(y-h-l K)^{2}}{2 \sigma^{2}}\right)
$$

with $y \in[-K / 2, K / 2)$. Finally, we obtain the Bhattacharyya constant as

$$
\begin{aligned}
\mathcal{B}\left(P_{L}\right)= & \int_{-K / 2}^{K / 2} \frac{1}{\sqrt{2 \pi \sigma^{2}}}\left(\sum_{l \in \mathbb{Z}} \exp \left(-\frac{(y-h-l K)^{2}}{2 \sigma^{2}}\right)\right) \\
& \times \exp \left(-\frac{y h}{\sigma^{2}}\right)\left(\sqrt{\frac{\sum_{l \in \mathbb{Z}} \exp \left(-\frac{l^{2} K^{2}-2 l(y+h) K}{2 \sigma^{2}}\right)}{\sum_{l \in \mathbb{Z}} \exp \left(-\frac{l^{2} K^{2}-2 l(y-h) K}{2 \sigma^{2}}\right)}}\right) d y,
\end{aligned}
$$

which can be computed numerically.

\section{REFERENCES}

[1] J. Wang, M. Ghosh, and K. Challapali, "Emerging cognitive radio applications: A survey," IEEE Commun. Mag., vol. 49, no. 3, pp. 74-81, Mar. 2011.

[2] S. Rini, D. Tuninetti, and N. Devroye, "Inner and outer bounds for the Gaussian cognitive interference channel and new capacity results," IEEE Trans. Inf. Theory, vol. 58, no. 2, pp. 820-848, Feb. 2012.

[3] R. K. Farsani, "On the capacity region of the broadcast, the interference, and the cognitive radio channels," IEEE Trans. Inf. Theory, vol. 61, no. 5, pp. 2600-2623, May 2015.

[4] R. K. Farsani and A. K. Khandani. (2017). Novel Techniques to Derive Capacity Results for Multi-User Interference Channels. [Online]. Available: https://arxiv.org/abs/1702.05846

[5] E. Kurniawan, A. Goldsmith, and S. Rini, "Practical coding schemes for cognitive overlay radios," in Proc. IEEE GLOBECOM, Anaheim, CA, USA, 2012, pp. 3760-3765.

[6] M. Shakiba-Herfeh, A. K. Tanc, and T. M. Duman, "LDPC code design for fast fading interference channels," in Proc. IEEE ICC, Kansas City, MO, USA, 2018, pp. 1-6.

[7] P. Berlin and D. Tuninetti, "LDPC codes for fading Gaussian broadcast channels," IEEE Trans. Inf. Theory, vol. 51, no. 6, pp. 2173-2182, Jun. 2005

[8] S. Sharifi, A. K. Tanc, and T. M. Duman, "Implementing the Han-Kobayashi scheme using low density parity check codes over Gaussian interference channels," IEEE Trans. Commun., vol. 63, no. 2, pp. 337-350, Feb. 2015.

[9] M. Uppal, G. Yue, Y. Xin, X. Wang, and Z. Xiong, "A robust multi-level design for dirty-paper coding," IEEE Trans. Commun., vol. 61, no. 7, pp. 2612-2623, Jul. 2013.

[10] M. W. Marcellin and T. R. Fischer, "Trellis coded quantization of memoryless and Gauss-Markov sources," IEEE Trans. Commun., vol. 38, no. 1, pp. 82-93, Jan. 1990. 